\title{
Uma Abordagem não Intrusiva para Reconhecimento de Atividades em Casas Inteligentes Explorando Processamento Semântico*
}

\author{
Eduardo Abreu ${ }^{1}$, Gerson Cavalheiro ${ }^{1}$, Ana Pernas ${ }^{1}$, Adenauer Yamin ${ }^{1}$, \\ João Lopes ${ }^{2}$, Cláudio Geyer ${ }^{3}$ \\ ${ }^{1}$ Universidade Federal de Pelotas (UFPEL) - Pelotas - RS - Brasil \\ ${ }^{2}$ Instituto Federal Sul-rio-grandense (IFSUL) - Pelotas - RS - Brasil \\ ${ }^{3}$ Universidade Federal do Rio Grande do Sul (UFRGS) - Porto Alegre - RS - Brasil \\ \{eabreu, gerson.cavalheiro, marilza, adenauer\}@inf.ufpel.edu.br \\ joaolopesecavg.ifsul.edu.br \\ geyerdinf.ufrgs.br
}

\begin{abstract}
The purpose of this work, named EXEHDA-AR (Execution Environment for Highly Distributed Applications-Activity Recognition), is to provide architectural features that allow the recognition of activities in the EXEHDA middleware, exploiting a semantic processing approach. For this purpose, architectural components were designed and integrated in the Subsystem of Adaptation and Context Recognition of EXEHDA. A case study about smart homes was developed to evaluate the functionalities proposed for the EXEHDA-AR, obtaining an average accuracy of $94.36 \%$ in the recognition of activities. These results indicate that methods based on semantic processing constitute a viable alternative, with low level of intrusion.
\end{abstract}

Resumo. O objetivo deste trabalho, denominado EXEHDA-AR (Execution Environment for Highly Distributed Applications-Activity Recognition), é prover recursos arquiteturais que permitam o reconhecimento de atividades no middleware EXEHDA, explorando uma abordagem baseada em processamento semântico. Para tanto foram concebidos componentes arquiteturais, os quais foram integrados ao Subsistema de Reconhecimento de Contexto e Adaptação do EXEHDA. Um estudo de caso sobre casas inteligentes foi desenvolvido para avaliar as funcionalidades propostas para o EXEHDA-AR, sendo obtida uma acurácia média de 94,36\% no reconhecimento de atividades. Estes resultados apontam que métodos baseados em processamento semântico constituem uma alternativa viável, com baixo nível de intrusão.

\section{Introdução}

Uma das aplicações do reconhecimento de atividades está no domínio ubíquo da $e$-Health, o qual pode ser explorado em sistemas de reabilitação, gerenciamento de doenças crônicas e monitoramento de idosos. Esse interesse na área de reconhecimento de atividades tem tido como motivação o envelhecimento da população, o que resulta em significativos desafios socioeconômicos no setor da saúde [Al-Shaqi et al. 2016].

\footnotetext{
*Trabalho realizado com apoio da CAPES (Programa Nacional de Cooperação Acadêmica- Procad) e da FAPERGS (Programa Pesquisador Gaúcho - PqG)
} 
Nesta perspectiva, uma alternativa é a utilização de casas inteligentes que proporcionem um ambiente assistido de vivência no contexto da saúde. Nestas residências devem ser contemplados serviços computacionais que possam auxiliar as pessoas nas suas práticas diárias, da forma mais transparente possível [Röcker et al. 2014]. Busca-se assim integrar a tecnologia ao cotidiano do usuário, caracterizando uma infraestrutura computacional de natureza ubíqua que deve exigir o menor envolvimento possível das pessoas no seu gerenciamento, bem como deve possibilitar que as atividades sejam reconhecidas a partir de dados de contexto coletados por sensores, os quais podem estar presentes em objetos carregados pelas pessoas ou incorporados ao ambiente em que as mesmas interagem [Perera et al. 2015].

Este trabalho, denominado EXEHDA-AR (Execution Environment for Highly Distributed Applications-Activity Recognition), contribui com o Subsistema de Reconhecimento de Contexto e Adaptação do middleware EXEHDA [Lopes et al. 2014], contemplando uma abordagem que permite o reconhecimento de atividades em casas inteligentes. Para tanto, explora métodos baseados em processamento semântico, sendo concebidos componentes arquiteturais integrados ao EXEHDA, bem como um modelo ontológico. O principal diferencial do EXEHDA-AR, quando comparado com os trabalhos relacionados, é a exploração do potencial de middleware para reconhecimento não intrusivo de atividades em intervalos de tempo próximos ao momento em que as rotinas estão sendo observadas.

\section{EXEHDA-AR: Visão Geral e Funcionalidades}

A proposta do EXEHDA-AR contempla os seguintes aspectos na sua concepção: (i) ser distribuída, permitindo a coleta e processamento das informações contextuais em diferentes localizações simbólicas; (ii) ser dirigida por eventos, possibilitando a associação de regras aos contextos de interesse das aplicações; e (iii) viabilizar a utilização de técnicas baseadas em especificação de regras para o reconhecimento de atividades. A premissa buscada no EXEHDA-AR é explorar de forma sinérgica a integração destes aspectos no suporte ao reconhecimento de atividades.

O ambiente ubíquo gerenciado pelo EXEHDA é constituído por células nas quais se distribuem os seguintes tipos de dispositivos computacionais: (i) EXEHDAbase: elemento central da célula responsável por todos serviços básicos; (ii) EXEHDAnodo: corresponde aos dispositivos computacionais responsáveis pela execução das aplicações; (iii) EXEHDAnodo móvel: tipo específico de EXEHDAnodo que corresponde aos dispositivos móveis; e (iv) EXEHDAborda: elemento de borda do ambiente ubíquo, responsável por fazer a interoperação entre os serviços do middleware e o ambiente.

O middleware EXEHDA possui dois tipos de servidores. O Servidor de Borda que é responsável por interagir com ambiente através de sensores e atuadores e o Servidor de Contexto que provê funcionalidades para ciência de contexto. Estes servidores são mapeados sobre o ambiente ubíquo, sendo o Servidor de Borda instanciado em equipamentos do tipo EXEHDAborda, enquanto o Servidor de Contexto é alocado no EXEHDAbase da célula.

$\mathrm{Na}$ proposta do EXEHDA-AR o middleware tem sua arquitetura estendida estendida com a inclusão de componentes para prover reconhecimento de atividades. De modo mais específico, a abordagem para reconhecimento de atividades no EXEHDA-AR 
tem por base o Servidor de Contexto e as funcionalidades de armazenamento e processamento das informações de contexto providas por este. Uma visão geral da arquitetura do Servidor de Contexto é apresentada na Figura 1.

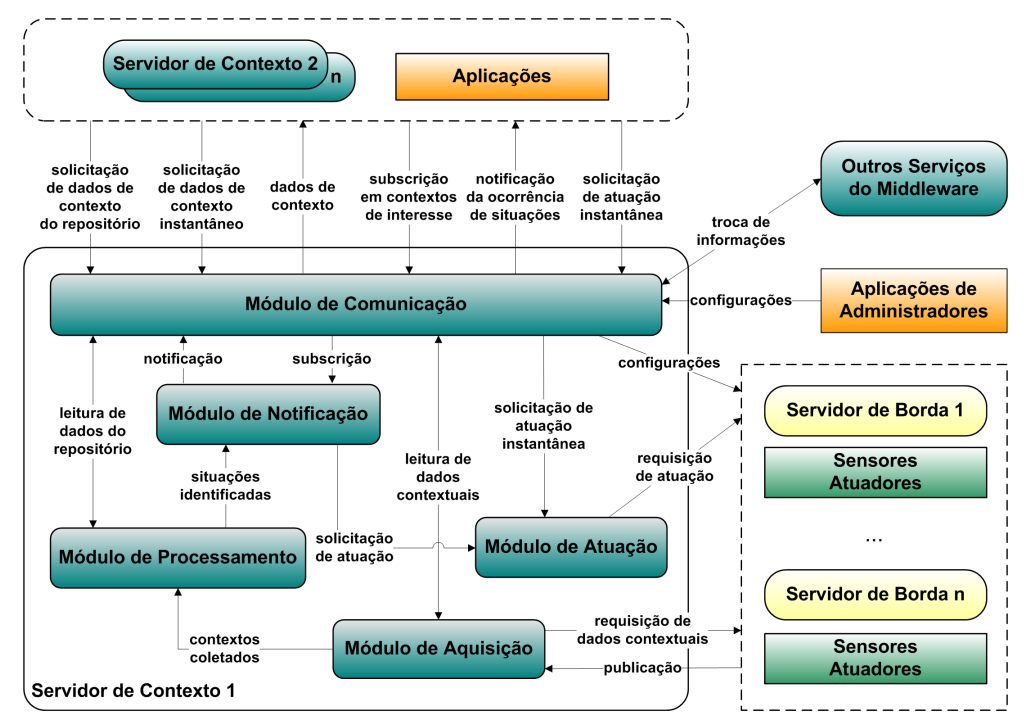

Figura 1. Servidor de Contexto

Os componentes para reconhecimento de atividades concebidos para o EXEHDA-AR foram introduzidos nos módulos de Aquisição e Processamento do Servidor de Contexto. Estes módulos, na perspectiva do EXEHDA-AR, são discutidos nas próximas seções.

\subsection{Módulo de Aquisição}

Responsável pelo suporte à captura dos dados contextuais coletados pelos Servidores de Borda, provendo suporte tanto para uma coleta agendada, como para a obtenção instantânea por solicitações de consumidores interessados.

Para o reconhecimento de atividades considerou-se necessário analisar o tempo da sequência de eventos, descrevendo-os como discretos para identificar padrões de interesse. Assim, o modelo de janelas deslizantes utilizado no EXEHDA-AR considerou o trabalho de [Salfner et al. 2010] que possui dois tipos janela: (i) baseada em uma quantidade específica de número de eventos que corresponde a um padrão de interesse; e (ii) janela baseada em tempo, que tem um tempo finito onde eventos de interesse são monitorados.

Como as atividades em uma casa inteligente se apresentam de forma dinâmica, foi concebido um algoritmo de janelamento de tempo finito. Este algoritmo foi implementado em Python com o objetivo de agrupar informações coletadas, oferecendo opções de aumentar ou diminuir a janela de tempo de acordo com perfil do morador da casa. Uma vez realizado o janelamento, as informações passam para o Módulo de Processamento.

\subsection{Módulo de Processamento}

Realiza as tarefas pertinentes ao tratamento das informações contextuais para identificar atividades relacionadas aos contextos de interesse das aplicações. Na Figura 2 é apresentada uma visão geral do Módulo de Processamento do EXEHDA-AR. 


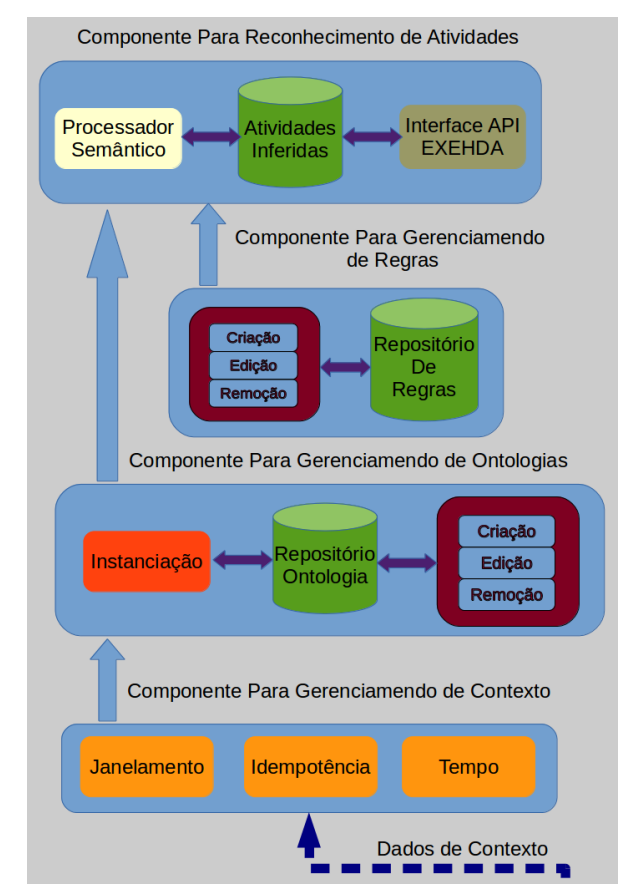

Figura 2. Módulo de Processamento

O Módulo de Processamento é constituído por quatro componentes, cujas funcionalidades são descritas a seguir.

\section{Componente para Gerenciamento de Contexto}

Responsável pelas as etapas de interpretação e agregação das informações contextuais. Estas etapas tem como objetivo aumentar o grau de abstração para melhorar a disponibilidade e usabilidade no reconhecimento de atividades.

Uma das formas de abstração providas por esse componente é a idempotência, a qual independentemente do número de vezes que sensor detecta eventos, é considerado que mesmo identificou uma ocorrência em uma janela de tempo válida. Outra abstração provida por este componente é a normalização do tipo de dado date time, cujas informações são convertidos para milissegundos, com intuito de serem utilizados na construção das regras.

Neste componente são agregadas informações, como identificação do sensor, o horário de início e fim e a identificação da janela em que foi coletado o evento.

\section{Componente para Gerenciamento de Ontologias}

Gerencia a camada semântica do Módulo de Processamento, possibilitando a instanciação de ontologias. A ontologia concebida para o reconhecimento de atividades no EXEHDA-AR, apresentada na Figura 3, utiliza como base a ontologia do projeto PalSPOT [Riboni et al. 2011], devido esta ser também instanciada em um middleware e focada no reconhecimento de atividades. Foram herdadas da ontologia original as classes Activity, Location, Artifact, Time e Person, das quais foram criadas subclasses, seus atributos e relacionamentos. Também, foi criada a classe Windows com a finalidade de receber as informações do Componente para Gerenciamento de Contexto. 
No EXEHDA-AR quando uma atividade é reconhecida, é criada uma nova instância na classe Activity relacionando-a com as demais classes para agregar informações relevantes como localização, objeto, segmento do dia e a janela em que a atividade foi reconhecida.

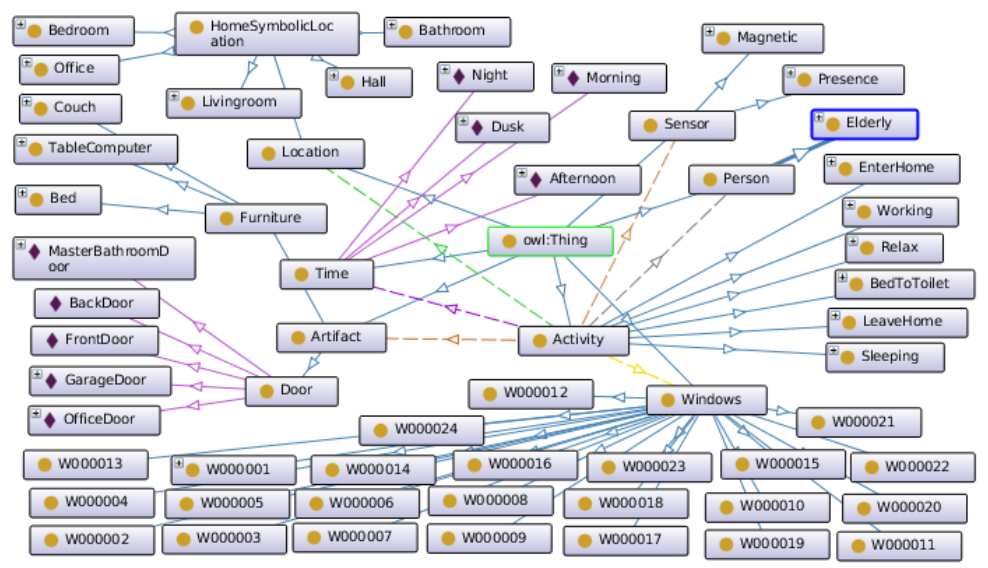

Figura 3. Visão Geral da Ontologia

A classe Activity tem subclasses com as atividades que se deseja reconhecer, como Sleeping, Working, EnterHome, LeaveHome e BedToToilet. Cada vez que uma atividade é reconhecida, o EXEHDA-AR cria uma instância na classe correspondente com as informações do tempo inicial e final da janela, objetos, localização, indivíduo e sensores.

As propriedades de dados recebem os valores oriundos dos sensores. Por exemplo, hasStatusSensor recebe o valor "dt" quando há eventos detectados. Devido a utilização da idempotência e do janelamento dos eventos de sensores apenas, recebe o valor de tempo do primeiro e do último evento na janela corrente.

A ontologia empregada pelo EXEHDA-AR é armazenada no Repositório de Ontologia e gerenciada por meio da API JENA [Riboni and Bettini 2011].

\section{Componente para Gerenciamento de Regras}

As regras são construídas em SWRL (Semantic Web Rule Language) [Okeyo et al. 2014], utilizando os conceitos propostos por [Chen and Nugent 2009], através da relação entre a localização simbólica e objeto para reconhecer qual atividade o indivíduo está realizando. Essas regras são processadas em nível de instâncias e, desta forma, motores de inferência conseguem raciocinar sobre as informações contextuais. As regras são armazenadas no Repositório de Regras, possibilitando ao desenvolvedor a criação, edição e remoção das mesmas.

Considerando que as regras estão associadas aos objetos do ambiente e a localização na casa, a partir dessas relações é possível identificar a atividade que está sendo realizado pelo usuário.

\section{Componente para Reconhecimento de Atividade}

Disponibiliza as funcionalidades para o processo de raciocínio sobre os dados contextuais, visando o reconhecimento das atividades. Neste componente as regras realizam a 
inferência nas instâncias da classe Windows, criando novas instâncias nas classes referentes às atividades através da função swrlx:makeOWLThing(?y, ?x).

Uma instância é criada quando a regra para reconhecer a atividade tem suas condições atendidas, sendo as informações relacionadas à instância criada, tais como: localização, objeto, sensores envolvidos na atividade.

\section{EXEHDA-AR: Estudo de Caso}

Para realização da avaliação do EXEHDA-AR foi selecionado um dataset pertencente ao grupo de pesquisa CASAS ${ }^{1}$, Universidade de Washington. Este dataset é proveniente de uma casa denominada Aruba, na qual uma senhora idosa reside sozinha, apresentando características alinhadas com as motivações do EXEHDA-AR.

Para modelar as regras de descrição lógica empregadas na avaliação do EXEHDA-AR foram escolhidas cinco atividades alvo: entrar em casa, sair de casa, dormir, ir da cama para banheiro e trabalhar no escritório. A linguagem SWRL foi utilizada para a elaboração das regras, sendo utilizados métodos descritos no trabalho [Chen and Nugent 2009]. O estudo de caso foi segmentado em cinco cenários que correspondem cada um deles a uma das atividades alvo que se busca reconhecer. $\mathrm{Na}$ próxima seção é apresentado o cenário "dormir". A descrição dos demais cenários pode ser encontrada em [Abreu 2017].

\subsection{Atividade "dormir"}

Maria dirige-se para o quarto, passa pela porta e deita-se na cama. Para inferir essa atividade considera-se que a localização neste cenário é o quarto, o objeto de interação é a cama (representada pelo sensor M003) e a interação da pessoa com objeto deve ser maior que 120000 milissegundos (20 minutos). Com isso, detecta-se que a pessoa está dormindo. Na Figura 4 é mostrada a distribuição dos sensores do quarto.

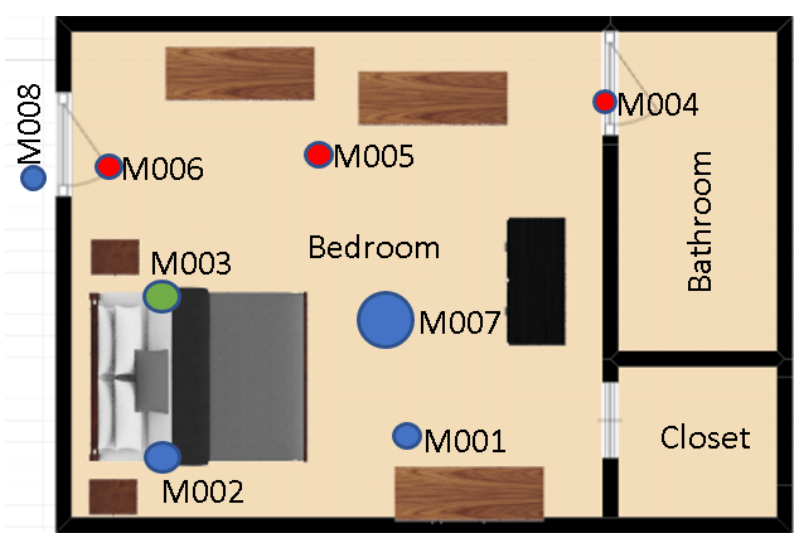

Figura 4. Sensores do quarto

Após observar a distribuição dos sensores pelo quarto, foi definido que o contexto de interesse está associado a detecção de presença pelo sensor M003. Por sua vez, os sensores M005, M006, M004 não devem detectar presença, assim indicando que pessoa permanece na cama. Na Figura 5 é possível observar a regra que foi elaborada para reconhecer a atividade "dormir" na casa Aruba.

\footnotetext{
${ }^{1}$ http://casas.wsu.edu/
} 


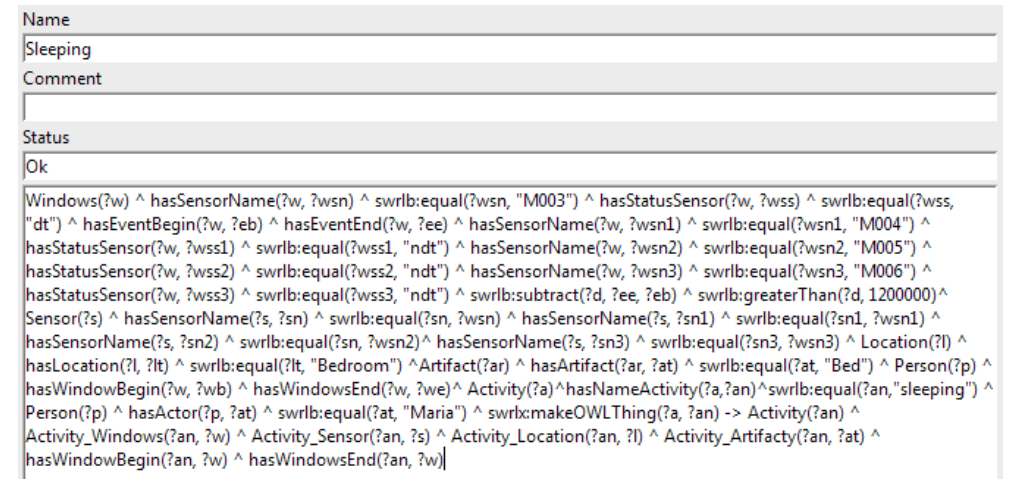

Figura 5. Regra em SWRL para atividade "dormir"

Utilizando essa regra, o EXEHDA-AR consegue reconhecer 398 das 401 ocorrências registradas na documentação do dataset, obtendo nessa atividade uma acurácia de 99,25\%. Após a realização das inferências é possível realizar uma busca semântica nos dados através da linguagem SPARQL, para obter mais informações sobre a atividade reconhecida. O resultado da consulta SPARQL mostra diferentes informações referentes a atividade "dormir", tais como: localização, objeto, o ator da atividade, sensores envolvidos, nome da atividade e a instância da janela na qual a atividade foi reconhecida.

\subsection{Avaliação de Acurácia no EXEHDA-AR}

Para avaliação da acurácia no EXEHDA-AR foi aplicado o método desenvolvido por [van Kasteren et al. 2011]. O método consiste em criar um matriz de confusão na qual as linhas representam atividades e as colunas a frequência dos verdadeiros positivos das atividades reconhecidas. A partir da matriz de confusão é possível mensurar a acurácia do reconhecimento das atividades. A seguinte fórmula apresenta uma visão geral do método: Accuracy $=\frac{\sum_{i=1}^{Q} T P_{i}}{\text { Total }}$

TP representa o número de verdadeiros positivos das atividades reconhecidas, $\mathbf{Q}$ o número de atividades a serem reconhecidas (dormir, entrar em casa...) e Total representa o número total de janelas de tempo deslizantes no conjunto de dados.

Assim, considerando esse método, foi construída uma matriz de confusão, mostrada na Figura 6, para avaliar a acurácia do EXEHDA-AR. Esta matriz apresenta uma estrutura de $5 \times 5$ colunas, onde cada cédula com fundo amarelo representa o número de atividades reconhecidas. As atividades estão representadas por números de 1 a 5 . A última coluna Ac/Atv representa a acurácia por atividade e a última linha a acurácia média do EXEHDA-AR. A representação das atividades estão descritas a seguir: (1) "dormir" tem 401 verdadeiros positivos; (2) "cama para banheiro" tem 157 verdadeiros positivos; (3) "entrar em casa" tem 431 verdadeiros positivos; (4) "sair de casa" tem 431 verdadeiros positivos; e (5) "trabalhar no escritório" tem 171 verdadeiros positivos.

Foi atingida uma acurácia média de 94,36\%, sendo que a atividade "dormir" teve o melhor resultado, enquanto que a atividade "trabalhar no escritório" apresentou um percentual menor de acurácia. Acurácia obtida pelo EXEHDA-AR mostrou-se promissora frente a trabalhos encontrados na revisão do estado da arte como [Cook 2012], [Riboni et al. 2011], [Van Kasteren et al. 2008] e [Tapia et al. 2004]. 


\begin{tabular}{|c|c|c|c|c|c|c|}
\hline Atividade & 1 & 2 & 3 & 4 & 5 & Ac/Atv \\
\hline 1 & 398 & - & - & - & - & $99,25 \%$ \\
\hline 2 & - & 154 & - & - & - & $98,08 \%$ \\
\hline 3 & - & - & 400 & - & - & $92.80 \%$ \\
\hline 4 & - & - & - & 405 & - & $93,96 \%$ \\
\hline 5 & - & - & - & - & 150 & $87,71 \%$ \\
\hline
\end{tabular}

Figura 6. Matriz de confusão do EXEHDA-AR

\section{Trabalhos Relacionados}

Tendo como base as premissas que motivaram o desenvolvimento desta pesquisa, os seguintes trabalhos foram selecionados: (I) Context-Aware System Infrastructure for Monitoring [Ni et al. 2016]; (II) AAL Domain Ontology for Event-based Human Activity Recognition [Culmone et al. 2014]; (III) Activity Recognition using ContextAware Infrastructure Ontology in Smart Home Domain [Wongpatikaseree et al. 2012]; e (IV) Semantic Smart Homes: Towards Knowledge Rich Assisted Living Environments [Chen et al. 2009].

A Tabela 1 mostra uma comparação entre os trabalhos relacionados e o EXEHDA-AR. Para esta comparação foram considerados os seguintes critérios: (i) proposição de um modelo semântico específico; (ii) forma de avaliação do modelo; (iii) verificação da acurácia; (iv) exploração de conceitos de objeto e localização; e (v) tipo de regra empregado para o reconhecimento de atividades.

Tabela 1. Comparação dos Trabalhos Relacionados

\begin{tabular}{c|c|c|c|c|c}
\hline $\begin{array}{c}\text { Trabalhos } \\
\text { Relacionados }\end{array}$ & $\begin{array}{c}\text { Propõe Modelo On- } \\
\text { tológico }\end{array}$ & $\begin{array}{c}\text { Utiliza } \\
\text { dataset }\end{array}$ & $\begin{array}{l}\text { Verifica a } \\
\text { Acurácia }\end{array}$ & $\begin{array}{l}\text { Explora Objeto e } \\
\text { Localização }\end{array}$ & $\begin{array}{l}\text { Tipo de Re- } \\
\text { gras }\end{array}$ \\
\hline I & Baseado em Modelo Existente & Não & Não & Parcialmente & JENA \\
\hline II & Baseado em Modelo Existente & Não & Não & Parcialmente & JENA \\
\hline III & Propõe Modelo Próprio & Não & Não & Utiliza & JENA \\
\hline IV & Propõe Modelo Próprio & Não & Não & Utiliza & AXIOMA \\
\hline EXEHDA-AR & Baseado em Modelo Existente & Sim & Sim & Utiliza & JENA e SWRL \\
\hline
\end{tabular}

O EXEHDA-AR tem o seu modelo ontológico baseado na ontologia PalSPOT. Por sua vez, os trabalhos I e II, são baseados nas ontologias DOLCE e DnS combinadas, sendo estas ontologias empregadas tanto para o reconhecimento de atividades como no gerenciamento da própria infraestrutura do ambiente inteligente. No caso do EXEHDA-AR, o gerenciamento do ambiente é feito por serviços do middleware EXEHDA. Os trabalhos III e IV desenvolveram seus modelos apenas utilizando os conceitos de localização e objeto, sendo que o trabalho IV emprega somente uma ontologia assim como EXEHDA-AR. Já o trabalho III utiliza duas ontologias uma para gerir o contexto da casa inteligente e outra para reconhecer atividades.

O EXEHDA-AR utiliza um dataset público para avaliar sua proposta, com dados de contextos coletados em um ambiente real, enquanto os trabalhos simulam atividades sem que as mesmas tenham sido coletadas de um ambiente real. O trabalho III faz uso de um framework chamado DB2R para criar suas ontologias a partir de uma base de dados relacional, realizando o reconhecimento analisando os $\log s$ de uma casa inteligente por meio de regras de descrição, o que o deixa próximo a abordagem do EXEHDA-AR. 
No EXEHDA-AR a coleta dos dados ocorre através do Servidor de Borda do middleware EXEHDA. De acordo com o dado coletado e a natureza da aplicação do usuário, os dados podem ser convertidos através de regras para um nível maior de abstração. Os demais trabalhos teriam que criar esses conceitos na ontologia ou ontologias como é caso do II e III, para realizar essa conversão e abstrair o contexto para nível mais alto.

Outro diferencial do EXEHDA-AR em relação aos trabalhos relacionados é que o EXEHDA-AR verifica a acurácia com relação à inferência em atividades diárias, uma vez que esta medida representa a qualidade do modelo ontológico frente aos desafios no reconhecimento de atividades humanas por meio de sensores não intrusivos.

\section{Considerações Finais}

A principal contribuição deste trabalho é a concepção dos componentes arquiteturais integrados ao middleware EXEHDA, os quais são responsáveis pelo reconhecimento de atividades, utilizando processamento semântico. A proposta do EXEHDA-AR, empregando sensores associados ao ambiente, e não diretamente no usuário, mostrou-se oportuna para a detecção de atividades, não necessitando que o usuário se envolva de forma intencional com o procedimento de monitoramento das suas atividades, caracterizando assim uma abordagem não intrusiva.

Os resultados do estudo de caso realizado no domínio de uma casa inteligente utilizando dados reais, mostraram-se promissores com uma acurácia média de 94,36\% de reconhecimento de atividade.

Dentre os aspectos levantados para continuidade do trabalho de pesquisa destacam-se: (i) desenvolver um modelo híbrido para reconhecimento de atividades, empregando técnicas baseadas em aprendizagem de máquina e em processamento semântico; (ii) viabilizar o reconhecimento de atividades oportunístico direcionado a atender a dinamicidade usual dos ambientes de IoT, quanto a entrada e saída de recursos de sensoriamento; e (iii) explorar o emprego dos mecanismos concebidos para o EXEHDA-AR em outros cenários de uso.

\section{Referências Bibliográficas}

Abreu, E. (2017). Reconhecimento de Atividades em Casas Inteligentes: Uma abordagem não Intrusiva explorando Processamento Semântico. Dissertação (mestrado em ciência da computação), Programa de Pós-Graduação em Computação, Centro de Desenvolvimento Tecnológico, Universidade Federal de Pelotas, Pelotas, RS.

Al-Shaqi, R., Mourshed, M., and Rezgui, Y. (2016). Progress in ambient assisted systems for independent living by the elderly. SpringerPlus, 5(1):624.

Chen, L. and Nugent, C. (2009). Ontology-based activity recognition in intelligent pervasive environments. International Journal of Web Information Systems, 5(4):410-430.

Chen, L., Nugent, C., Mulvenna, M., Finlay, D., and Hong, X. (2009). Semantic smart homes: towards knowledge rich assisted living environments. In Intelligent Patient Management, pages 279-296. Springer.

Cook, D. J. (2012). Learning setting-generalized activity models for smart spaces. IEEE intelligent systems, 27(1):32-38. 
Culmone, R., Falcioni, M., Giuliodori, P., Merelli, E., Orru, A., Quadrini, M., Ciampolini, P., Grossi, F., and Matrella, G. (2014). Aal domain ontology for event-based human activity recognition. In Mechatronic and Embedded Systems and Applications (MESA), 2014 IEEE/ASME 10th International Conference on, pages 1-6. IEEE.

Lopes, J. L., de Souza, R. S., Geyer, C., da Costa, C., Barbosa, J., Pernas, A. M., and Yamin, A. (2014). A middleware architecture for dynamic adaptation in ubiquitous computing. J. UCS, 20(9):1327-1351.

Ni, Q., García Hernando, A. B., and Pau de la Cruz, I. (2016). A context-aware system infrastructure for monitoring activities of daily living in smart home. Journal of Sensors, 2016.

Okeyo, G., Chen, L., Wang, H., and Sterritt, R. (2014). Dynamic sensor data segmentation for real-time knowledge-driven activity recognition. Pervasive and Mobile Computing, 10:155-172.

Perera, C., Member, C. H. L., Jayawardena, S., and Chen, M. (2015). Context-aware computing in the internet of things: A survey on internet of things from industrial market perspective. arXiv preprint arXiv:1502.00164.

Riboni, D. and Bettini, C. (2011). Cosar: hybrid reasoning for context-aware activity recognition. Personal and Ubiquitous Computing, 15(3):271-289.

Riboni, D., Pareschi, L., Radaelli, L., and Bettini, C. (2011). Is ontology-based activity recognition really effective? In Pervasive Computing and Communications Workshops (PERCOM Workshops), 2011 IEEE International Conference on, pages 427-431. IEEE.

Röcker, C., Ziefle, M., and Holzinger, A. (2014). From computer innovation to human integration: current trends and challenges for pervasive healthtechnologies. In Pervasive Health, pages 1-17. Springer.

Salfner, F., Lenk, M., and Malek, M. (2010). A survey of online failure prediction methods. ACM Computing Surveys (CSUR), 42(3):10.

Tapia, E. M., Intille, S. S., and Larson, K. (2004). Activity recognition in the home using simple and ubiquitous sensors. In International Conference on Pervasive Computing, pages $158-175$. Springer.

Van Kasteren, T., Noulas, A., Englebienne, G., and Kröse, B. (2008). Accurate activity recognition in a home setting. In Proceedings of the 10th international conference on Ubiquitous computing, pages 1-9. ACM.

van Kasteren, T. L., Alemdar, H., and Ersoy, C. (2011). Effective performance metrics for evaluating activity recognition methods. ARCS 2011.

Wongpatikaseree, K., Ikeda, M., Buranarach, M., Supnithi, T., Lim, A. O., and Tan, Y. (2012). Activity recognition using context-aware infrastructure ontology in smart home domain. In Knowledge, Information and Creativity Support Systems (KICSS), 2012 Seventh International Conference on, pages 50-57. IEEE. 